\section{Hospitalizações por causas respiratórias e cardiovasculares associadas à contaminação atmosférica no Município de São Paulo, Brasil}

\author{
Respiratory and cardiovascular hospitalizations \\ associated with air pollution in the city of \\ São Paulo, Brazil
}

\footnotetext{
${ }^{1}$ Faculdade de Medicina, Universidade de São Paulo, São Paulo, Brasil.

2 Centro de Vigilância Epidemiológica, Secretaria de Estado da Saúde, São Paulo, Brasil.

Correspondência N. Gouveia Departamento de Medicina Preventiva, Faculdade de Medicina, Universidade de São Paulo.

Av. Dr. Arnaldo 455, São Paulo, SP 01246-903, Brasil. ngouveia@usp.br
}

\begin{abstract}
The recognition that current air pollution levels cause harmful health effects makes the definition of the air quality regulatory process imperative. This study examines the association between exposure to air pollution and hospital admissions in the city of São Paulo, aiming to support the development of measures to reduce such health risks. The authors conducted an ecological time series study of hospital admissions for respiratory and cardiovascular diseases in children and the elderly in relation to daily air pollution levels, using generalized additive Poisson regression models. All air pollutants except ozone showed a statistically significant association with admissions for respiratory and cardiovascular diseases. An increase of $10 \mu \mathrm{g} / \mathrm{m} 3$ in fine particulate matter was associated with a $4.6 \%$ increase in asthma admissions in children and a $4.3 \%$ increase in admissions for chronic obstructive pulmonary disease and $1.5 \%$ for ischemic heart disease in the elderly. These associations are consistent with a large body of literature in this area and indicate that the current air pollution levels in São Paulo have an important negative impact on the population's health.
\end{abstract}

Air Pollution; Morbidity; Respiratory Tract Diseases; Cardiovascular Diseases

\author{
Nelson Gouveia 1 \\ Clarice Umbelino de Freitas 2 \\ Lourdes Conceição Martins 1 \\ Izabel Oliva Marcilio ${ }^{1}$
}

\section{Introdução}

Apesar dos notáveis avanços obtidos nas últimas décadas em direção a um ambiente com ar mais limpo, especialmente nos países desenvolvidos, os atuais níveis de poluição experimentados pela maior parcela da população humana continuam a se mostrar danosos à saúde. Os primeiros estudos relacionando a poluição atmosférica e implicações na saúde datam da metade do século passado, e ainda hoje essa relação é observada e comprovada por trabalhos de investigação científica.

Inúmeros estudos têm encontrado associações significantes entre níveis diários de material particulado inalável com diâmetro $\leq 10 \mu \mathrm{m}$ $\left(\mathrm{PM}_{10}\right)$, monóxido de carbono (CO), ozônio $\left(\mathrm{O}_{3}\right)$ e outros poluentes e uma série de efeitos na saúde que vão desde mortalidade total na população 1,2,3,4, passando por adoecimentos para causas específicas 5,6,7,8,9,10,11, até malformações congênitas 12 ou menor ganho de peso durante a gestação 13 .

As inúmeras evidências que indicam que a poluição do ar em nosso meio é suficiente para causar danos à saúde e a demanda pela definição de processos reguladores referentes aos padrões de qualidade do ar tornam imprescindíveis o melhor detalhamento dessa associação, com a identificação de grupos populacionais especiais, patologias específicas e níveis ambientais nos quais o processo exposição-adoecimen- 
to ou morte acontecem. Dessa forma, informações provenientes de investigações sistemáticas com dados gerados localmente são de grande importância para subsidiar o planejamento e avaliação de programas de saúde voltados a essa questão 14,15,16

No Brasil, o Ministério da Saúde está implantando o programa de Vigilância dos Efeitos na Saúde Relacionados à Poluição do Ar (VIGIAR). Desse modo, realizou-se o presente estudo que busca analisar a associação entre exposição à poluição do ar e internações hospitalares para causas específicas de doenças respiratórias e cardiovasculares em idosos e crianças no Município de São Paulo. Este trabalho foi desenvolvido no âmbito do Projeto de Estruturação do Sistema Nacional de Vigilância em Saúde do Sistema Único de Saúde (VIGISUS) e foi realizado na maior metrópole brasileira, que conta com competentes serviços de monitoramento da qualidade do ar, dispõe de estatísticas de morbidade de qualidade adequada e, sobretudo, possui grande contingente populacional exposto a níveis de poluição do ar potencialmente prejudiciais à saúde. Seu objetivo principal é fornecer subsídios para a elaboração de medidas que visem reduzir os riscos à saúde associados à poluição do ar.

\section{Métodos}

Foram utilizadas as bases de dados referentes ao Sistema de Informações Hospitalares do SUS. Todas as internações de idosos (pessoas com 65 anos ou mais) e crianças (menores de 5 anos) que ocorreram no período entre lo de maio de 1996 e 31 de abril de 2000, em hospitais do Município de São Paulo foram extraídas dessa base de dados. As doenças investigadas em cada faixa etária estão descritas no Tabela 1 .
Informações sobre níveis diários de material particulado $\left(\mathrm{PM}_{10}\right)$, monóxido de carbono (CO), dióxido de enxofre $\left(\mathrm{SO}_{2}\right)$, dióxido de nitrogênio $\left(\mathrm{NO}_{2}\right)$ e ozônio $\left(\mathrm{O}_{3}\right)$ foram obtidas junto à Companhia de Tecnologia de Saneamento Ambiental (CETESB). A CETESB dispõe de 14 estações fixas em São Paulo. Para todos os poluentes a coleta de dados compreendeu um período de 24 horas, que se iniciava à primeira hora do dia. Para o $\mathrm{PM}_{10}$ e $\mathrm{SO}_{2}$ foi calculada a média aritmética de 24 horas, para o $\mathrm{NO}_{2}$ e $\mathrm{O}_{3}$ foi utilizada a máxima de 24 horas e para o CO foi utilizada a maior média móvel de 8 horas para cada uma das estações de monitoramento. A seguir, foi calculada a média aritmética por dia entre as estações de monitoramento. Assumiu-se que esta estimativa era representativa da poluição do ar no Município de São Paulo ${ }^{8}$. Informações sobre temperatura mínima e umidade relativa do ar foram obtidas junto ao Instituto de Ciências Astronômicas e Geofísicas, Universidade de São Paulo (IAG/USP).

Para caracterizar a relação existente entre os diversos poluentes e entre esses e os fatores meteorológicos foram calculados coeficientes de correlação de Pearson.

O número diário de internações para cada um dos desfechos descritos na Tabela 1 foi considerado como variáveis dependentes, e os níveis médios diários dos poluentes atmosféricos foram analisados como variáveis independentes. As variáveis de controle introduzidas nos modelos foram: dia da semana (variável indicadora), número de dias transcorridos (função não paramétrica de alisamento), fatores meteorológicos (termos lineares ou funções não paramétricas de alisamento), e feriados (variável indicadora).

A variável "dia da semana" foi utilizada para controlar a sazonalidade de curta duração. Para controlar a sazonalidade de longa duração, foi utilizada a variável "número de dias transcorri-

Tabela 1

Desfechos estudados, detalhados por capítulos da Classificação Internacional de Doenças-10ạ Revisão (CID-10), por faixa etária e números totais observados. São Paulo, Brasil, 1996-2000.

\begin{tabular}{lccc}
\hline Desfecho & CID-10 & Faixa etária (anos) & Totais \\
\hline Doenças do aparelho respiratório & J00-J99 & $<5$ & 54.860 \\
& & $\geq 65$ & 12.509 \\
Pneumonias & J12-J18 & $\geq 5$ & 33.227 \\
& & $<5$ & 5.093 \\
Asma & J45 & $\geq 65$ & 10.480 \\
Doença pulmonar obstrutiva crônica & J40-J47 & $\geq 65$ & 2.884 \\
Doenças do aparelho circulatório & I00-199 & $\geq 65$ & 33.816 \\
Doença isquêmica do coração & I20-122, I24 & 6.885 \\
\hline
\end{tabular}


dos”, ou seja, uma variável numérica, crescente, representando o número total de dias entre o início do estudo e um determinado dia. Como forma de minimizar o efeito da variabilidade devida ao acaso (ruído branco) para esta variável trabalhou-se com uma função não paramétrica, o loess ou alisador móvel de regressão 17. O loess permite controlar uma dependência não linear entre a variável de interesse (neste caso, internações) e a sazonalidade. Para temperatura e umidade, foram empregados termos lineares ou funções de alisamento de acordo com cada modelo. Diferentes defasagens foram testadas para cada variável de forma a minimizar o Critério de Informação de Akaike (AIC) 18. Foram utilizados também termos auto-regressivos de 1a ou 2a ordem para ajustar os modelos para correlação serial entre os dados 19 .

Utilizaram-se modelos aditivos generalizados (GAM) 19 em regressão de Poisson com ajuste para sobredispersão. Os GAM são modelos que permitem a utilização simultânea de termos paramétricos e não paramétricos. Neste estudo assumiu-se uma relação linear entre os poluentes e as internações.

As manifestações biológicas dos efeitos da poluição sobre a saúde apresentam, aparentemente, uma defasagem em relação à exposição do indivíduo aos agentes poluidores. Os atendimentos observados em um dia específico podem tanto estar relacionados à poluição do referido dia, como ao da poluição observada em dias anteriores. Os estudos na literatura variam quanto ao número de dias de defasagem avaliados. Neste trabalho, testaram-se os valores diários dos poluentes em diferentes defasagens, bem como as médias móveis de dois a cinco dias. Optou-se por apresentar aqui os resultados mais estatisticamente significantes (positivos ou negativos) do efeito de cada poluente nos diversos desfechos.

Foram feitas análises dos resíduos padronizados por meio das funções de autocorrelação (ACF) e funções de autocorrelação parciais (PACF) de cada modelo de regressão, assim como observado o Critério de Informação de Akaike para se verificar o ajuste de cada modelo final. Adotou-se o nível de significância $\alpha=5 \%$ em todas as análises.

Os coeficientes obtidos dos modelos de regressão foram transformados por exponenciação em riscos relativos (RR) de hospitalização para um incremento de $10 \mu \mathrm{g} / \mathrm{m}^{3}$ ou $1 \mathrm{ppm}$ nos níveis médios diários dos poluentes ambientais. Os resultados são também apresentados como aumento percentual nas internações, que são obtidos dos RR pela fórmula:

Aumento \% $=(\mathrm{RR}-1) * 100$
Todas as análises foram realizadas com o programa estatístico S-Plus (Mathsoft Inc., Seattle, Estados Unidos).

\section{Resultados}

Podemos observar pela Tabela 2 que as médias do $\mathrm{PM}_{10}$ e do $\mathrm{NO}_{2}$ ultrapassaram os padrões anuais de qualidade do ar $\left(50 \mu \mathrm{g} / \mathrm{m}^{3}\right.$ e $100 \mu \mathrm{g} / \mathrm{m}^{3}$, respectivamente) no período estudado, o que não ocorreu com o $\mathrm{SO}_{2}$. Na observação dos dados ano a ano, verificou-se que o $\mathrm{O}_{3}$ apresentou níveis crescentes, ao contrário dos demais poluentes (dado não apresentado). Em relação às internações registradas, pode-se observar que as doenças circulatórias em idosos vêm em primeiro lugar, seguidas das doenças respiratórias em crianças. Dentre estas, pneumonia foi o diagnóstico mais freqüente. Vale ressaltar que as internações respiratórias em crianças (Tabela 1) correspondem a cerca de $30 \%$ do total de internações observadas no período nessa faixa etária, enquanto as hospitalizações por doença isquêmica do coração em idosos representam aproximadamente $14 \%$ das internações observadas nesse grupo.

Tanto os níveis diários dos poluentes, com destaque para o material particulado inalável $\left(\mathrm{PM}_{10}\right)$, quanto o número diário de internações para as várias causas, apresentaram um padrão sazonal bastante característico, com maiores valores nos meses de inverno (Figura 1).

A Tabela 3 apresenta os coeficientes de correlação de Pearson dos poluentes entre si e em relação às variáveis meteorológicas. Pode-se notar que todos os poluentes estão estatisticamente correlacionados entre si, exceto o $\mathrm{CO}$ com $\mathrm{O}_{3}$. As maiores correlações são observadas entre o $\mathrm{PM}_{10}$ e $\mathrm{NO}_{2}, \mathrm{CO}$ e $\mathrm{SO}_{2}$. A temperatura mínima está inversamente correlacionada com o $\mathrm{PM}_{10}, \mathrm{SO}_{2}, \mathrm{CO}$ e $\mathrm{NO}_{2}$. Encontrou-se correlação inversa entre a umidade relativa do ar e todos os poluentes.

A Tabela 4 apresenta o risco relativo ajustado de internações por doenças respiratórias em crianças para incrementos nos níveis diários de poluição. Na análise dos dados, observou-se que todos os poluentes apresentaram associação positiva com as internações por doenças respiratórias totais e os subgrupos asma e pneumonia em praticamente todas as defasagens, porém os resultados estatisticamente mais significantes foram observados com a média móvel de 5 dias. $\mathrm{O} \mathrm{O}_{3}$ foi o que apresentou associações menos consistentes com as internações em crianças.

De maneira geral os maiores riscos foram observados para internações por asma. Por exemplo, um aumento de $10 \mu \mathrm{g} / \mathrm{m}^{3}$ no nível diário de $\mathrm{PM}_{10}$ esteve associado a um aumento de $2,4 \%$ 
Estatística descritiva dos níveis de poluição, variáveis meteorológicas e das internações de idosos e crianças. São Paulo, Brasil, $1996-2000$.

\begin{tabular}{|c|c|c|c|c|c|c|c|c|}
\hline Parâmetros & n (dias) & Média & DP & $\begin{array}{c}\text { Valor } \\
\text { mínimo }\end{array}$ & $25 \%$ & $50 \%$ & $75 \%$ & $\begin{array}{c}\text { Valor } \\
\text { máximo }\end{array}$ \\
\hline $\mathrm{PM}_{10}\left(\mu \mathrm{g} / \mathrm{m}^{3}\right)$ & 1.461 & 54,49 & 25,24 & 13,93 & 37,04 & 48,57 & 64,56 & 186,25 \\
\hline $\mathrm{SO}_{2}\left(\mu \mathrm{g} / \mathrm{m}^{3}\right)$ & 1.459 & 17,71 & 9,86 & 2,58 & 10,54 & 15,38 & 22,83 & 75,24 \\
\hline $\mathrm{CO}$ (ppm) & 1.459 & 3,24 & 1,64 & 0,54 & 2,17 & 2,86 & 3,87 & 12,58 \\
\hline $\mathrm{NO}_{2}\left(\mu \mathrm{g} / \mathrm{m}^{3}\right)$ & 1.452 & 103,46 & 51,18 & 19,00 & 68,05 & 93,73 & 127,97 & 421,62 \\
\hline $\mathrm{O}_{3}\left(\mu \mathrm{g} / \mathrm{m}^{3}\right)$ & 1.460 & 71,79 & 41,99 & 7,82 & 42,41 & 61,85 & 91,69 & 389,45 \\
\hline Temperatura $\left({ }^{\circ} \mathrm{C}\right)$ & 1.461 & 19,21 & 3,45 & 7,13 & 16,70 & 19,15 & 22,01 & 28,34 \\
\hline Umidade relativa (\%) & 1.461 & 81,29 & 8,06 & 48,45 & 77,08 & 81,98 & 86,79 & 96,63 \\
\hline \multicolumn{9}{|l|}{ Internações em idosos } \\
\hline Respiratórias & 1.461 & 14,76 & 5,41 & 1 & 11 & 14 & 18 & 36 \\
\hline DPOC & 1.461 & 3,59 & 2,58 & 0 & 2 & 3 & 5 & 19 \\
\hline Pneumonias & 1.461 & 5,94 & 3,07 & 0 & 4 & 6 & 8 & 20 \\
\hline Circulatórias & 1.461 & 43,38 & 15,71 & 10 & 32 & 43 & 52 & 165 \\
\hline Doença isquêmica do coração & 1.461 & 8,60 & 3,83 & 0 & 6 & 8 & 11 & 21 \\
\hline \multicolumn{9}{|l|}{ Internações em crianças } \\
\hline Respiratórias & 1.461 & 37,77 & 14,73 & 7 & 27 & 36 & 46 & 95 \\
\hline Asma & 1.461 & 6,19 & 3,47 & 0 & 4 & 6 & 8 & 27 \\
\hline Pneumonias & 1.461 & 22,48 & 10,09 & 3 & 15 & 21 & 28 & 65 \\
\hline
\end{tabular}

DP = desvio padrão; DPOC = doença pulmonar obstrutiva crônica.

Figura 1

Níveis diários de material particulado inalável (PM) e número diário de internações por doença respiratória

em idosos (Resp_65). São Paulo, Brasil, 1996-2000.

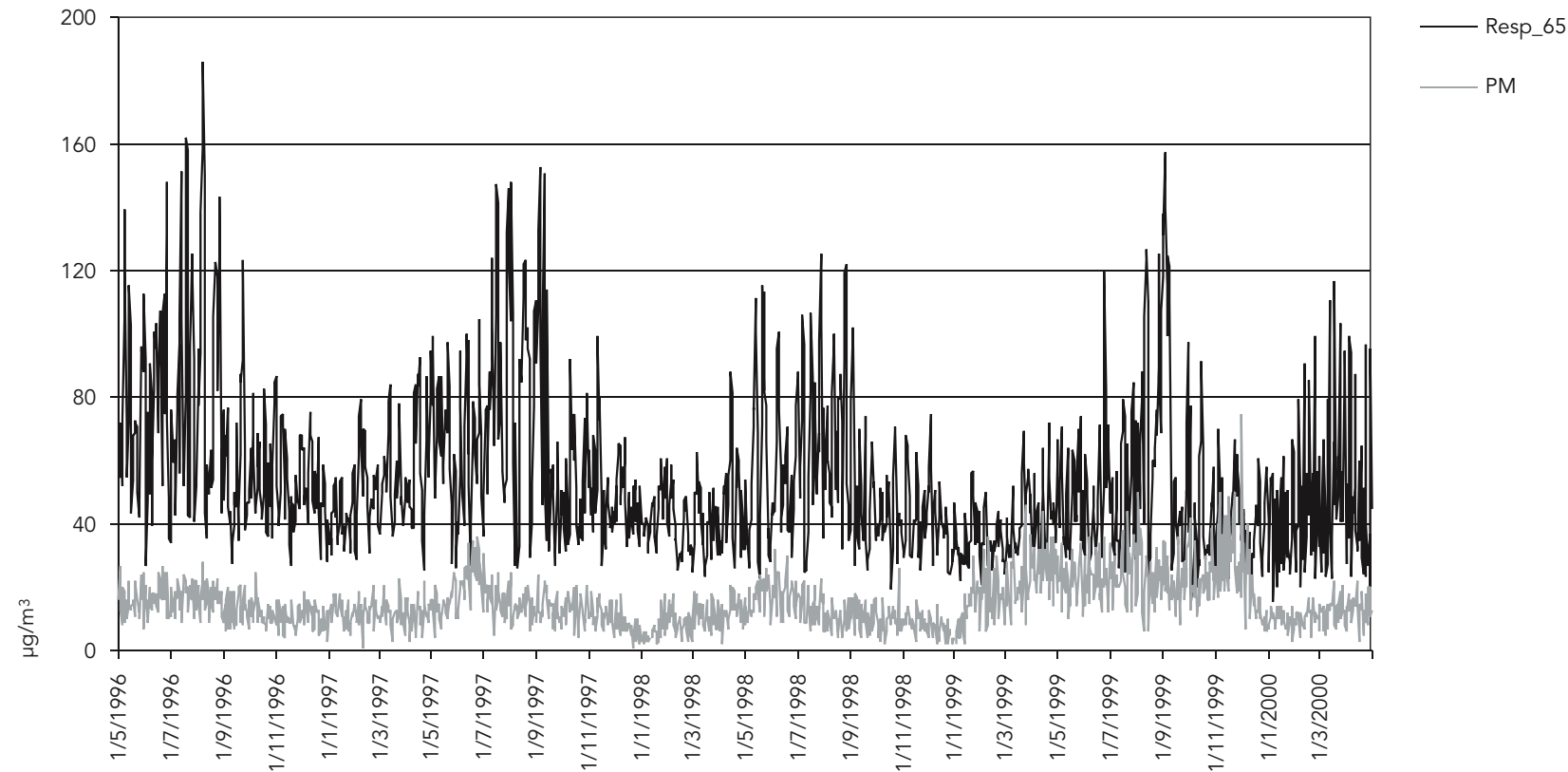


Coeficientes de correlação de Pearson entre os valores diários de poluição do ar e variáveis meteorológicas.

\begin{tabular}{|c|c|c|c|c|c|c|c|}
\hline & $\mathrm{PM}_{10}$ & $\mathrm{SO}_{2}$ & $\mathrm{CO}$ & $\mathrm{NO}_{2}$ & $\mathrm{O}_{3}$ & T (mínima) & UR (média) \\
\hline $\mathrm{PM}_{10}$ & 1,00 & & & & & & \\
\hline $\mathrm{SO}_{2}$ & 0,68 * & 1,00 & & & & & \\
\hline $\mathrm{CO}$ & $0,71 *$ & 0,56 * & 1,00 & & & & \\
\hline $\mathrm{NO}_{2}$ & 0,77 * & 0,67 * & 0,68 * & 1,00 & & & \\
\hline $\mathrm{O} 3$ & 0,29 * & $0,21 *$ & 0,00 & 0,32 * & 1,00 & & \\
\hline T (mínima) & $-0,33$ * & $-0,35$ * & $-0,19$ * & $-0,26$ * & 0,02 & 1,00 & \\
\hline UR (média) & $-0,48$ * & $-0,38$ * & $-0,37$ * & $-0,36$ * & $-0,33$ * & 0,07 & 1,00 \\
\hline
\end{tabular}

$\mathrm{T}=$ temperatura; $\mathrm{UR}=$ umidade relativa do ar.

${ }^{*} p<0,05$.

Tabela 4

Riscos relativos (RR) e intervalo de confiança de 95\% (IC95\%) de internações por doenças respiratórias em crianças para um incremento de $10 \mu \mathrm{g} / \mathrm{m}^{3}$ nos níveis de $\mathrm{PM}_{10}, \mathrm{SO}_{2}, \mathrm{NO}_{2}$ e $\mathrm{O}_{3}$ e de $1 \mathrm{ppm}$ para o CO. São Paulo, Brasil, 1996-2000.

\begin{tabular}{lccc}
\hline Doenças & Niveis & RR & IC95\% \\
\hline \multirow{2}{*}{ Doenças respiratórias } & $\mathrm{PM}_{10}$ & 1,024 & $1,017-1,031$ \\
& $\mathrm{SO}_{2}$ & 1,067 & $1,049-1,086$ \\
& $\mathrm{CO}$ & 1,019 & $1,007-1,030$ \\
& $\mathrm{NO}_{2}$ & $1,009-1,013$ & $1,003-1,012$ \\
Asma & $\mathrm{O}_{3}$ & 1,008 & $1,033-1,060$ \\
& $\mathrm{PM}_{10}$ & 1,046 & $1,072-1,148$ \\
Pneumonia & $\mathrm{SO}_{2}$ & 1,109 & $1,032-1,077$ \\
& $\mathrm{CO}$ & 1,054 & $1,015-1,030$ \\
& $\mathrm{NO}_{2}$ & 1,023 & $1,007-1,025$ \\
& $\mathrm{O}_{3}$ & 1,016 & $1,014-1,029$ \\
& $\mathrm{PM}_{10}$ & 1,021 & $1,033-1,074$ \\
& $\mathrm{SO}_{2}$ & 1,053 & $1,006-1,030$
\end{tabular}

nas internações de crianças menores de 5 anos por doenças respiratórias, de $2,1 \%$ por pneumonia e de $4,6 \%$ por asma.

Para as internações por doença respiratória em idosos, os riscos relativos e seus respectivos intervalos de confiança são apresentados na Tabela 4 . Novamente, todos os poluentes apresentaram associações estatisticamente significantes com as internações por doenças respiratórias totais e os subgrupos pneumonia e doença pulmonar obstrutiva crônica (DPOC), com exceção do $\mathrm{O}_{3}$ que não apresentou associação com as internações por pneumonia (Tabela 5).
As defasagens entre exposição e o evento internação também foram semelhantes às encontradas na análise para crianças menores de 5 anos, ou seja, os resultados mais significantes foram obtidos com a média móvel de 4 ou 5 dias para a maioria dos poluentes (sem defasagem para o CO e internações por doenças respiratórias e defasagem de 3 dias para $\mathrm{PM}_{10}$ e DPOC). Por outro lado, as internações por pneumonia mostraram uma relação muito mais imediata com resultados mais significantes quando a exposição foi sem defasagem, isto é, no mesmo dia do evento. 
Risco relativo (RR) e intervalo de confiança de 95\% (IC95\%) de internações por doenças respiratórias e cardiovasculares em idosos (65 anos ou mais) para um incremento de $10 \mu \mathrm{g} / \mathrm{m}^{3}$ nos níveis de $\mathrm{PM}_{10}, \mathrm{SO}_{2}, \mathrm{NO}_{2}$ e $\mathrm{O}_{3}$ e de 1 ppm para o CO. São Paulo, Brasil, 1996-2000.

\begin{tabular}{|c|c|c|c|}
\hline Doenças & Níveis & $\mathbf{R R}$ & IC95\% \\
\hline \multirow[t]{5}{*}{ Doenças Respiratórias } & $\mathrm{PM}_{10}$ & 1,022 & $1,014-1,031$ \\
\hline & $\mathrm{SO}_{2}$ & 1,113 & $1,088-1,139$ \\
\hline & $\mathrm{CO}$ & 1,024 & $1,013-1,035$ \\
\hline & $\mathrm{NO}_{2}$ & 1,012 & $1,007-1,017$ \\
\hline & $\mathrm{O}_{3}$ & 1,009 & $1,004-1,014$ \\
\hline \multirow[t]{5}{*}{ DPOC } & $\mathrm{PM}_{10}$ & 1,043 & $1,028-1,058$ \\
\hline & $\mathrm{SO}_{2}$ & 1,179 & $1,126-1,235$ \\
\hline & $\mathrm{CO}$ & 1,049 & $1,023-1,076$ \\
\hline & $\mathrm{NO}_{2}$ & 1,024 & $1,015-1,034$ \\
\hline & $\mathrm{O}_{3}$ & 1,015 & $1,005-1,025$ \\
\hline \multirow[t]{5}{*}{ Pneumonia } & $\mathrm{PM}_{10}$ & 1,019 & $1,007-1,030$ \\
\hline & $\mathrm{SO}_{2}$ & 1,087 & $1,055-1,119$ \\
\hline & $\mathrm{CO}$ & 1,039 & $1,022-1,057$ \\
\hline & $\mathrm{NO}_{2}$ & 1,008 & $1,002-1,014$ \\
\hline & $\mathrm{O}_{3}$ & 1,005 & 0,999-1,011 \\
\hline \multirow[t]{4}{*}{ Doenças circulatórias } & $\mathrm{PM}_{10}$ & 1,010 & $1,005-1,015$ \\
\hline & $\mathrm{SO}_{2}$ & 1,033 & $1,019-1,046$ \\
\hline & $\mathrm{CO}$ & 1,016 & $1,008-1,023$ \\
\hline & $\mathrm{NO}_{2}$ & 1,005 & $1,002-1,007$ \\
\hline \multirow[t]{4}{*}{ Doença isquêmica do coração } & $\mathrm{PM}_{10}$ & 1,015 & $1,005-1,025$ \\
\hline & $\mathrm{SO}_{2}$ & 1,071 & $1,049-1,095$ \\
\hline & $\mathrm{CO}$ & 1,016 & $1,002-1,030$ \\
\hline & $\mathrm{NO}_{2}$ & 1,006 & $1,002-1,010$ \\
\hline
\end{tabular}

DPOC = doença pulmonar obstrutiva crônica.

Um aumento de $10 \mu \mathrm{g} / \mathrm{m}^{3}$ nos níveis de $\mathrm{PM}_{10}$ esteve associado a um incremento nas hospitalizações em idosos da ordem de $2,2 \%$ para doenças respiratórias, de 4,3\% para DPOC e de 1,9\% para pneumonia. O aumento de 1 ppm nos níveis de CO correspondeu à elevação no número de hospitalizações de idosos em $2,4 \%$ para doenças respiratórias, de 4,9\% para DPOC e de 3,9\% para pneumonia.

Para as internações por doenças do aparelho circulatório e mais especificamente doenças isquêmicas do coração, encontraram-se associações estatisticamente significantes para todos os poluentes nas diversas defasagens, com exceção do $\mathrm{O}_{3}$ (Tabela 5). Para hospitalizações por doenças circulatórias, diferentemente das doenças respiratórias, observaram-se resultados mais significantes nas hospitalizações sem defasagem entre exposição e evento. Para as doenças isquêmicas do coração, a defasagem mais significante foi a média móvel de 2 dias.
Um aumento de $10 \mu \mathrm{g} / \mathrm{m}^{3}$ nos níveis de $\mathrm{PM}_{10}$ esteve associado a um incremento nas hospitalizações em idosos por doenças do aparelho circulatório de $1 \%$ e de $1,5 \%$ para doenças isquêmicas do coração.

\section{Discussão}

Este estudo procurou, por intermédio de análises de séries temporais, detalhar a relação entre exposição à poluição atmosférica e a ocorrência de hospitalizações por doenças cardiovasculares e respiratórias em crianças e idosos na cidade de São Paulo.

Os poluentes mais associados aos vários desfechos e à magnitude dos efeitos estão de acordo com a literatura nacional 6,7,8,9,10,14 e internacional 20,21,22 sobre o assunto. Observou-se uma associação estatisticamente significante entre o aumento no nível de poluentes na atmosfera e o 
aumento de hospitalizações pelas diversas causas, nos dois grupos etários estudados. Apesar da pequena magnitude dos RR encontrados, o impacto da poluição do ar na saúde da população deve ser substancial, levando-se em conta o grande número de indivíduos expostos.

Os resultados apresentados representam uma aproximação quantitativa do impacto da poluição atmosférica na saúde da população. É importante salientar que o desfecho aqui estudado, hospitalização, é apenas um dos muitos efeitos causados pela poluição do ar. Efeitos como a ocorrência de sintomas, uso de medicação e redução na atividade física, entre outros, não são avaliados no presente trabalho. Estes, considerados de menor gravidade para a saúde do indivíduo, são de grande importância para a saúde pública, dada a elevada freqüência com que ocorrem e devido ao impacto negativo na qualidade de vida das pessoas e de conseqüências econômicas negativas, como o absenteísmo escolar e no trabalho.

Este estudo utiliza dados secundários para o cálculo dos coeficientes e riscos relativos para hospitalizações decorrentes do aumento do nível de poluição atmosférica observada. Esses dados são provenientes de fontes estáveis e bastante confiáveis 11,23, sendo utilizados amplamente em trabalhos técnicos e científicos. É importante ressaltar que os registros são referentes a hospitalizações ocorridas na rede pública de saúde. Dessa forma, os resultados aqui apresentados refletem os efeitos da poluição do ar na parcela da população que utiliza esse serviço, que corresponde à maior parte da população do país.

Por outro lado, as informações do Sistema de Informações Hospitalares do SUS utilizadas neste estudo são produzidas com objetivos contábeis e não estritamente para estudos epidemiológicos, podendo, assim, apresentar algum grau de inacurácia. Pode ter havido ainda algum grau de subestimação da freqüência de determinados agravos, tendo em vista o perfil tecnológico da rede de assistência, além de erros na codificação dos diagnósticos. Um outro problema presente nessa base de dados é a possibilidade da dupla contagem de um mesmo paciente, uma vez que o sistema não identifica reinternações.

Contudo, em estudos ecológicos de séries temporais de base diária, fatores cuja distribuição não varia diariamente não atuam como possíveis variáveis de confusão 11. A qualidade das informações sobre internações hospitalares e outros possíveis problemas dessa base de dados não variam dia a dia e não estão correlacionados com os níveis de poluição do ar. Portanto, suas implicações sobre as estimativas de efeito encontradas são mínimas.
Por outro lado, há que se lembrar de que se trata de um estudo ecológico em que não se dispõe de informações individuais sobre exposição e doença. Por exemplo, não se pode afirmar com certeza que aqueles indivíduos que foram hospitalizados eram os mais expostos aos níveis de poluição do ar. Todavia, como a exposição é medida de maneira ecológica (pela média dos níveis mensurados em toda a cidade de São Paulo) assume-se que uma variação no nível médio de poluição do ar num determinado dia no Município reflita também uma variação na exposição média de cada indivíduo residente nele.

Uma outra questão que deve ser mencionada é o problema identificado no critério de convergência quando são utilizados modelos aditivos generalizados no programa S-Plus 24. Quando há mais do que uma função não paramétrica nesse tipo de análise e o parâmetro a ser estimado é pequeno, a convergência do modelo não fica assegurada e este pode produzir estimativas enviesadas dos coeficientes de regressão e de seus erros padrões. A maioria dos modelos em nossas análises, no entanto, possuía apenas uma função não paramétrica. Além disso, o viés identificado é de pequena magnitude e nem sempre ocorre 24 . Desse modo, as estimativas aqui apresentadas podem ser vistas como uma boa aproximação do efeito da poluição nas hospitalizações de crianças e idosos do Município de São Paulo.

A relação entre poluição atmosférica e doenças respiratórias vem sendo avaliada em diversos estudos, nacionais e internacionais, e os mecanismos fisiopatológicos envolvidos nessa relação têm sido bastante estudados. A relação entre doenças cardiovasculares e a poluição do ar, entretanto, foi menos explorada, e as bases fisiopatológicas dessa associação ainda não estão explicadas. Estudos sugerem dois possíveis mecanismos para explicar o prejuízo da condição cardiovascular pela ação dos poluentes. O primeiro envolve mudanças na modulação do sistema nervoso autônomo, efeito demonstrável pela observação da diminuição da variabilidade da freqüência cardíaca em relação aos níveis de poluição. Algumas patologias do sistema cardiovascular, como infarto agudo do miocárdio e morte súbita, estão associadas a alterações no sistema nervoso autônomo 25,26. O outro possível mecanismo está relacionado ao aumento de fibrinogênio e fatores de inflamação circulantes, fato que leva ao aumento da viscosidade sangüínea e propicia a coagulação. Tais fatores associados a eventos isquêmicos e exacerbação de doenças cardiovasculares pré-existentes 26 .

Estimar o risco para a saúde da população em razão da poluição do ar é um primeiro passo para o planejamento e implementação de ações vi- 
sando a um ambiente mais saudável. A produção de dados tecnicamente bem embasados é fundamental para a formulação de políticas públicas e tomada de decisões que promovam o desenvolvimento sócio-econômico e que levem em conta questões ambientais e a qualidade de vida das pessoas. Os resultados aqui apresentados indicam em termos quantitativos o risco para um au-

\section{Resumo}

A constatação de que os atuais níveis de poluição atmosférica são suficientes para causar danos à saúde torna imprescindível a definição de processos reguladores para a qualidade do ar. Este estudo analisa a associação entre exposição à poluição atmosférica e internações hospitalares no Município de São Paulo, Brasil, visando subsidiar a elaboração de medidas para redução dos riscos à saúde. Realizou-se um estudo ecológico de séries temporais, analisando hospitalizações por causas respiratórias e cardiovasculares em crianças e idosos em relação aos níveis diários observados de poluentes, por meio de modelos aditivos generalizados em regressão de Poison. Todos os poluentes, com exceção do ozônio, apresentaram associação significante com internações respiratórias e cardiovasculares. Um aumento de $10 \mu \mathrm{g} / \mathrm{m}^{3}$ no nível de material particulado inalável associa-se ao incremento de 4,6\% nas internações por asma em crianças, de 4,3\% por doença pulmonar obstrutiva crônica em idosos e de 1,5\% por doença isquêmica do coração também em idosos. Essas associações entre aumento no nível de poluentes na atmosfera e o aumento de hospitalizações estão de acordo com a literatura nacional e internacional sobre o assunto e indicam que os níveis atuais de contaminação do ar no Município de São Paulo têm impacto na saúde de sua população.

Poluição do Ar; Morbidade; Doenças Respiratórias; Doenças Cardiovasculares mento no número de hospitalizações de crianças e idosos de acordo com um aumento no nível de poluição na cidade de São Paulo. Espera-se que os dados apresentados sirvam como ferramentas para um melhor entendimento do impacto da poluição do ar na saúde das pessoas, colaborando para o monitoramento e saneamento dessa questão em São Paulo e em outras metrópoles.

\section{Colaboradores}

N. Gouveia planejou e desenhou o estudo, coordenou a execução e as análises, escreveu e revisou o manuscrito. C. U. Freitas e L. C. Martins contribuíram na coleta e organização dos dados, execução das análises, e na escrita e revisão do manuscrito. I. O. Marcilio escreveu e revisou o manuscrito. 


\section{Referência}

1. Conceição GM, Miraglia SG, Kishi HS, Saldiva $\mathrm{PH}$, Singer JM. Air pollution and child mortality: a time-series study in Sao Paulo, Brazil. Environ Health Perspect 2001; 109 Suppl 3:347-50.

2. Daumas RP, Mendonça GAS, Leon AP. Poluição do ar e mortalidade no Município do Rio de Janeiro: análise de série temporal. Cad Saúde Pública 2004; 20:311-9.

3. Gouveia N, Fletcher T. Time series analysis of air pollution and mortality: effects by cause, age and socioeconomic status. J Epidemiol Community Health 2000; 54:750-5.

4. Stieb DM, Judek S, Burnett RT. Meta-analysis of time-series studies of air pollution and mortality: effects of gases and particles and the influence of cause of death, age, and season. J Air Waste Manage Assoc 2002; 52:470-84.

5. Brilhante OM, Tambellini AM. Particulate suspended matters and cases of respiratory disease in Rio de Janeiro city (Brazil). Int J Environ Health Res 2002; 12:169-74.

6. Lin CA, Pereira LA, Conceição GMS, Kishi HS, Milani R, Braga ALF, et al. Association between air pollution and ischemic cardiovascular emergency room visits. Environ Res 2003; 92:57-63.

7. Martins LC, Latorre MR, Saldiva PH, Braga AL. Air pollution and emergency room visits due to chronic lower respiratory diseases in the elderly: an ecological time-series study in Sao Paulo, Brazil. J Occup Environ Med 2002; 44:622-7.

8. Braga ALF, Conceição GMS, Pereira LA, Kishi H, Pereira J, Andrade M. Air pollution and pediatric hospital admissions in São Paulo, Brazil. Journal of Environmental Medicine 1999; 1:95-102.

9. Martins LC, Latorre MR, Cardoso MR, Gonçalves FL, Saldiva PH, Braga AL. Air pollution and emergency room visits due to pneumonia and influenza in Sao Paulo, Brazil. Rev Saude Publica 2002; 36:88-94.

10. Braga AL, Saldiva PH, Pereira LA, Menezes JJ, Conceição GM, Lin CA, et al. Health effects of air pollution exposure on children and adolescentes in São Paulo, Brazil. Pediatr Pulmonol 2001; 31:106-13.

11. Gouveia N, Fletcher T. Respiratory diseases in children and outdoor air pollution in São Paulo, Brazil: a time series analysis. Occup Environ Med 2000; 57:477-83.

12. Ritz B, Yu F, Fruin S, Chapa G, Shaw GM, Harris JA. Ambient air pollution and risk of birth defects in Southern California. Am J Epidemiol 2002; 155:17-25.

13. Gouveia N, Bremner SA, Novaes HM. Association between ambient air pollution and birth weight in São Paulo, Brazil. J Epidemiol Community Health 2004; 58:11-7.

14. Gouveia N, Mendonça GAS, Ponce-de-Leon A, Correia JEM, Junger WL, Freitas CU, et al. Poluição do ar e efeitos na saúde nas populações de duas grandes metrópoles brasileiras. Epidemiol Serv Saúde 2003; 12:29-40.
15. Cifuentes L, Borja-Aburto VH, Gouveia N, Thurston G, Davis DL. Assessing the health benefits of urban air pollution reductions associated with climate change mitigation (2000-2020): Santiago, Sao Paulo, Mexico City, and New York City. Environ Health Perspect 2001; 109 Suppl 3:419-25.

16. The World Bank, United Nations Development Programme/World Bank Energy Sector Management Assistance Programme. Health effects of outdoor air pollution. South Asia Urban Air Quality Management Briefing Note N. 11. http://www.worldbank.org/sarurbanair (acessado em 20/Jan/2005).

17. Cleveland WS. Robust locally-weighted regression: an approach to regression analysis by local fitting. J Am Stat Assoc 1979; 83:597-610.

18. Akaike, H. Information theory and an extension of the maximum likelihood principle. In: Petrov BN, Csàki F, editors. Second International Symposium on Information Theory. Budapest: Akademiai Kiadó; 1973. p. 267-81.

19. Hastie T, Tibshirani R. Generalized additive models. London: Chapman and Hall; 1990.

20. Luginaah IN, Fung KY, Gorey KM, Webster G, Wills C. Association of ambient air pollution with respiratory hospitalization in a government-designated "area of concern": the case of Windsor, Ontario. Environ Health Perspect 2005; 113:290-6.

21. Atkinson RW, Anderson HR, Sunyer J, Ayres J, Baccini M, Vonk JM, et al. Acute effects of particulate air pollution on respiratory admissions: results from APHEA 2 project. Air pollution and health: a European approach. Am J Respir Crit Care Med 2001; 164 (10 Pt 1):1860-6.

22. Dominici F, Peng RD, Bell ML, Pham L, McDermott A, Zeger SL, et al. Fine particulate air pollution and hospital admission for cardiovascular and respiratory diseases. JAMA 2006; 295:1127-34.

23. Veras C, Martins M. A confiabilidade dos dados nos formulários de Autorização de Internação Hospitalar (AIH), Rio de Janeiro, Brasil. Cad Saúde Pública 1994; 10:339-55.

24. Dominici F, McDermott A, Zegger SL, Samet JM. On the use of generalized additive models in timeseries studies of air pollution and health. Am J Epidemiol 2002; 156:193-203.

25. Holguin F, Tellez-Rojo MM, Hernandez M, Cortez M, Chow JC, Watson JG, et al. Air pollution and heart rate variability among the elderly in Mexico City. Epidemiology 2003; 14:521-7.

26. Metzger KB, Tolbert PE, Klein M, Peel JL, Flanders WD, Todd K, et al. Ambient air pollution and cardiovascular emergency department visits. Epidemiology 2004; 15:46-56.
Recebido em 11/Jul/2005
Versão final reapresentada em 05/Abr/2006
Aprovado em 23/Mai/2006 